\title{
Chemical properties of rutherfordium (Rf) and dubnium (Db) in the aqueous phase
}

\author{
Yuichiro Nagame ${ }^{1, a}$, Jens Volker Kratz ${ }^{2}$, and Matthias Schädel ${ }^{3}$ \\ ${ }^{1}$ Advanced Science Research Center, Japan Atomic Energy Agency, Tokai, Ibaraki 319-1195, Japan \\ ${ }^{2}$ Institut für Kernchemie, Johannes Gutenberg-Universität Mainz, 55128 Mainz, Germany \\ ${ }^{3}$ GSI Helmholtzzentrum für Schwerionenforschung GmbH, 64291 Darmstadt, Germany
}

\begin{abstract}
Recent experimental studies of the chemical characterization of the first two transactinide elements, rutherfordium (Rf) and dubnium $(\mathrm{Db})$, conducted atom-at-a-time in aqueous phases are reviewed. A short description on experimental techniques based on partition methods, specifically automated rapid chemical separation systems, is also given. Perspectives for aqueous-phase chemistry experiments on heavier elements are briefly discussed.
\end{abstract}

\section{Introduction}

Chemical studies of the transactinide elements with atomic numbers $Z \geq 104$ provide not only crucial and challenging opportunities to advance our understanding of properties of matter at the limits of existence but they also elucidate the influence of relativistic effects on atomic electrons and the architecture of the Periodic Table of the Elements at the farthest reach [1-6]. These elements are all man-made ones synthesized at accelerators using nuclear reactions of heavy-ion beams with heavy element target materials and they can only be identified by measuring their characteristic nuclear decay, or that of their known daughter nuclei, with sensitive detection techniques. As both half-lives and cross sections of these nuclides are rapidly decreasing with increasing $Z$, they are usually available in quantities of only a few atoms or often one atom-at-a-time.

The chemical characterization of the transactinide elements in aqueous-phase experiments has been accomplished by partition methods with single atoms, e.g. liquid-liquid extraction, ion-exchange chromatography, and reversed-phase extraction chromatography. The ultimate goal of these atom-at-a-time scale partition experiments is to determine the socalled distribution coefficient, $K_{\mathrm{d}}$, as a function of ligand concentration. The $K_{\mathrm{d}}$ value is given in its simplest definition as the ratio of the number of atoms - determined by its radioactivity either in the organic phase (in liquid-liquid extractions) or in the stationary phase (in column chromatography) to that in the aqueous phase. In order to get statistically significant results, it is indispensable to repeat the same experimental procedure several hundred or even several thousand times with cycle times of about the lifetime of the nuclide under investigation. In these processes, the behaviour of the transactinide element is compared with that of its lighter homologs under identical conditions. Investigations have been carried out with automated rapid chemical separation apparatuses to characterize chemical properties of these elements.

\footnotetext{
${ }^{a}$ e-mail: nagame.yuichiro@jaea.go.jp
} 
Here we summarize recent studies of the chemical separation and characterization of the first two transactinide elements, rutherfordium (Rf) and dubnium (Db), the only ones which so far have been investigated in greater detail in aqueous phases. Some perspectives for further studies are briefly given. Large parts of this paper are based on previous review articles [7-10].

\section{Aqueous-phase experiments}

Extensive series of detailed investigations were made possible by the development of computer-controlled automated systems that have greatly improved the ability to perform rapidly and reproducibly large numbers of chromatographic separations on miniaturized columns. This made it possible to systematically vary ligand concentrations to determine the stoichiometry of the eluted species. The experiments with automated devices have produced detailed and sometimes surprising results that called for a detailed theoretical modelling of the chemical properties with improved relativistic quantum-chemical calculations. Recent experimental results obtained with automated rapid chemical separation systems are summarized in [10].

The aqueous-phase experiments with the transactinide elements are carried out on the basis of the following steps: (i) synthesis of a specific isotope of a transactinide element, (ii) rapid transport of this nuclide to chemical separation devices by an aerosol loaded gas-jet transport technique, (iii) fast chemical characterization that includes dissolution in an aqueous solution containing inorganic ligands for complex formation, (iv) partition of this complex between the aqueous and an organic phase or an ion-exchange resin, (v) preparation of a sample suitable for nuclear spectroscopy ( $\alpha$-particle spectroscopy), which usually requires the evaporation of an aqueous solution to dryness, and (vi) detection of nuclides through their characteristic nuclear decay properties for an unambiguous identification. For characterization of the transactinide elements, recent partition experiments have been conducted using the automated rapid ion-exchange separation apparatus ARCA II (Automated Rapid Chemistry Apparatus) with a miniaturized computer-controlled liquid chromatography system [11] and AIDA (Automated Ion-exchange separation apparatus coupled with the Detection system for Alpha spectroscopy) [12]; AIDA consists of ARCA II and an automated on-line $\alpha$-particle detection system. AIDA enables cyclic discontinuous column chromatographic separations of short-lived nuclides in aqueous phases and automated detection of $\alpha$ particles within a typical cycle time of $60 \mathrm{~s}$.

\section{Element 104, rutherfordium (Rf)}

Pioneering aqueous-phase experiments with 68 -s ${ }^{261} \mathrm{Rf}$ produced in the ${ }^{248} \mathrm{Cm}\left({ }^{18} \mathrm{O}, 5 n\right)$ reaction clearly indicated that the behaviour of $\mathrm{Rf}$ is fundamentally different from that of heavy actinides $[13,14]$. Although these results were key experiments demonstrating that a new transition element series, the $6 d$-electron series, begins with $Z=104$, none of these experiments provided detailed information on Rf chemistry; no $K_{\mathrm{d}}$ values were measured in these first-generation experiments.

Recent systematic investigations of the chromatographic behaviour of Rf performed atom-at-a-time showed that the properties of $\mathrm{Rf}$ are similar to those of the group-4 homologs, zirconium ( $\mathrm{Zr}$ ) and hafnium (Hf), although there are sometimes striking differences in the complexation strength between Rf and the lighter homologs. These results confirm that $\mathrm{Rf}$ is a member of the group-4 elements in the Periodic Table [7-10]. On the contrary, fluoride complex formation of $\mathrm{Rf}$ is significantly different from that of the homologs $\mathrm{Zr}$ and 

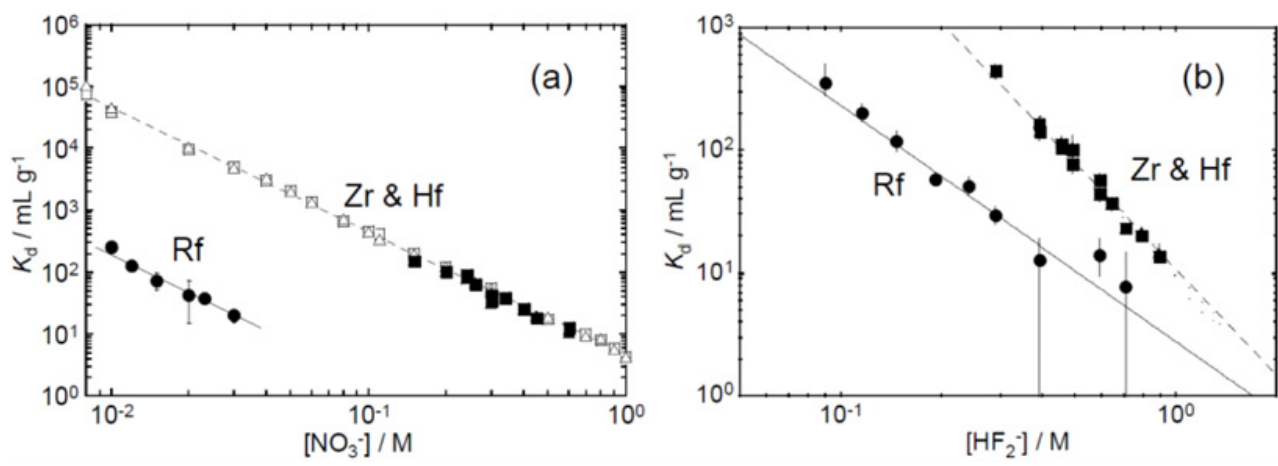

Figure 1. (a) Distribution coefficients $\left(K_{\mathrm{d}}\right)$ of $\mathrm{Zr}$ and $\mathrm{Hf}$ measured under static conditions (open symbols) and those of $\mathrm{Zr}$, Hf, and Rf from column chromatography (solid symbols) as a function of the equilibrated concentration of $\mathrm{NO}_{3}^{-},\left[\mathrm{NO}_{3}^{-}\right]$, at fixed $\left[\mathrm{F}^{-}\right]=3.0 \times 10^{-3} \mathrm{M}$. Linear relationships of the $\log K_{\mathrm{d}}$ vs. $\log \left[\mathrm{NO}_{3}^{-}\right]$for Rf and the homologs are indicated by the solid and dashed lines, respectively [17]. (b) Distribution coefficients $\left(K_{\mathrm{d}}\right)$ for $\mathrm{Zr}$, Hf, and Rf on the anion-exchange resin as a function of the $\mathrm{HF}_{2}^{-}$concentration, $\left[\mathrm{HF}_{2}^{-}\right]$. Adapted from $[17,18]$.

Hf, demonstrating a surprising behaviour of Rf [15-18]. Here, we summarize the fluoride complex formation studies of Rf performed with ARCA II and AIDA.

The successive fluoride complex formation of cationic species of $\mathrm{Zr}$, Hf, and $\mathrm{Rf}$ was investigated through cation-exchange chromatography with ARCA II at the Philips Cyclotron, Paul Scherrer Institute [15] and with AIDA at the JAEA tandem accelerator [1618]. The results demonstrated that the distribution coefficient, $K_{\mathrm{d}}$, of Rf in mixed solution of $\mathrm{HF} / 0.10 \mathrm{M} \mathrm{HNO}_{3}$ decreases with increasing concentration of the fluoride ion, $\left[\mathrm{F}^{-}\right]$, indicating the consecutive formation of neutral and/or anionic fluoride complexes of $\mathrm{Rf}$ as those of homologs. It was also ascertained that at these low $\mathrm{F}^{-}$concentrations the fluoride complex formation of $\mathrm{Rf}$ is significantly weaker than that of $\mathrm{Zr}$ and $\mathrm{Hf}$. The sequence of adsorption strength on the cation-exchange resin is $\mathrm{Zr} \leq \mathrm{Hf}<\mathrm{Rf}$ at any given $\left[\mathrm{F}^{-}\right][15,16]$. The observed $K_{\mathrm{d}}$ sequence among the group- 4 element complexes is in agreement with that predicted theoretically in reference [19]. Here, free energy changes of the complex formation reactions were determined on the basis of fully relativistic density functional theory calculations of various hydrated and hydrolysed fluoride complexes of $\mathrm{Zr}$, Hf, and Rf.

Anionic fluoride complexation has been studied in mixed $\mathrm{HF} / \mathrm{HNO}_{3}$ solutions (dilute HF concentration) [17]. Figure 1a shows the variation of the $K_{\mathrm{d}}$ values for $\mathrm{Zr}$, Hf and Rf as a function of the counter anion $\mathrm{NO}_{3}^{-},\left[\mathrm{NO}_{3}^{-}\right]$, at a constant $\left[\mathrm{F}^{-}\right]$of $3.0 \times 10^{-3} \mathrm{M}$. It is found that the $K_{\mathrm{d}}$ values of $\mathrm{Zr}$ and $\mathrm{Hf}$ are identical and the logarithmic values of $K_{\mathrm{d}}$ linearly decrease with the logarithmic increase of $\left[\mathrm{NO}_{3}^{-}\right]$with a slope of $-2.0 \pm 0.1$, as indicated by the dashed line. The $K_{\mathrm{d}}$ values of $\mathrm{Rf}$ are indicated by closed circles. As shown in Fig. 1a, at any given $\left[\mathrm{NO}_{3}^{-}\right]$the $K_{\mathrm{d}}$ values of $\mathrm{Rf}$ are much smaller than those of $\mathrm{Zr}$ and $\mathrm{Hf}$, and they smoothly decrease with increasing $\left[\mathrm{NO}_{3}^{-}\right]$with the slope of $-2.2 \pm 0.2$, as indicated by the solid line. The results indicate that, at constant $\left[\mathrm{F}^{-}\right]$of $3.0 \times 10^{-3} \mathrm{M}$, anionic complexes of $\mathrm{Zr}$, Hf, and $\mathrm{Rf}$ are present as $\left[\mathrm{MF}_{6}\right]^{2-}(\mathrm{M}=\mathrm{Zr}$, Hf, and $\mathrm{Rf})$ [17]. The formation of an anionic fluoride complex of Rf was interpreted in detail by taking into account not only chemical equilibria of anion-exchange reactions and of formation reactions of fluoride complexes but also comparing those with the ones of the homologs $\mathrm{Zr}$ and Hf. Upper limits of formation constants for the fluoride complexes of Rf were experimentally evaluated for the first time. 
The result demonstrated that the formation of the hexafluoride complex of $\mathrm{Rf},\left[\mathrm{RfF}_{6}\right]^{2-}$, is at least one order of magnitude smaller than those of the homologs $\mathrm{Zr},\left[\mathrm{ZrF}_{6}\right]^{2-}$, and $\mathrm{Hf}$, $\left[\mathrm{HfF}_{6}\right]^{2-}[17]$.

In addition, also anion-exchange experiments in HF solution (higher HF concentration) showed surprising results [18]. Figure 1(b) depicts the $K_{\mathrm{d}}$ values for $\mathrm{Zr}$, Hf, and $\mathrm{Rf}$ as a function of $\mathrm{HF}_{2}^{-}$concentration, $\left[\mathrm{HF}_{2}^{-}\right]$. The $K_{\mathrm{d}}$ values of the group-4 elements decrease linearly with $\left[\mathrm{HF}_{2}^{-}\right]$in a $\log K_{\mathrm{d}}$ vs. $\log \left[\mathrm{HF}_{2}^{-}\right]$plot. It should be noted here that the slopes for $\mathrm{Zr}$ and $\mathrm{Hf}$ are clearly -3 (dashed line), while that for $\mathrm{Rf}$ is significantly different, i.e. -2 (solid line). This implies that $\mathrm{Rf}$ is likely to be present as the hexafluoride complex, $\left[\mathrm{RfF}_{6}\right]^{2-}$, similar to the well-known $\left[\mathrm{ZrF}_{6}\right]^{2-}$ and $\left[\mathrm{HfF}_{6}\right]^{2-}$ at lower concentration of $\mathrm{HF}$, while $\mathrm{Zr}$ and $\mathrm{Hf}$ are likely to be present in the form of the heptafluoride complexes, $\left[\mathrm{ZrF}_{7}\right]^{3-}$ and $\left[\mathrm{HfF}_{7}\right]^{3-}$. This shows a strikingly different fluoride complexing behaviour between $\mathrm{Rf}$ and its homologs. As mentioned above, the experimental finding on the fluoride complexation sequence $\mathrm{Zr} \geq \mathrm{Hf}>\mathrm{Rf}$ is in qualitative agreement with relativistic molecular density functional calculations [19].

The fast centrifuge system SISAK (Short-lived Isotopes Studied by the AKUFVEtechnique, where AKUFVE is a Swedish acronym for an arrangement of continuous investigations of distribution ratios in liquid extraction) was applied to perform continuous liquid-liquid extraction and detection of short-lived Rf using small-volume centrifuges and a liquid-scintillation detection system. SISAK, employed to extract 4 -s ${ }^{257} \mathrm{Rf}$ produced in the ${ }^{208} \mathrm{~Pb}\left({ }^{50} \mathrm{Ti}, n\right)$ reaction, was coupled to the recoil separator BGS (Berkeley Gas-filled Separator) at the Lawrence Berkeley National Laboratory (LBNL) 88-inch cyclotron [20]. Recoiling ${ }^{257} \mathrm{Rf}$, pre-separated from the primary beam and nuclear reaction by-products, was thermalized in a stopping-gas cell and was transferred to SISAK through the aerosol loaded gas-jet system. The extraction behaviour of $\mathrm{Rf}$ was similar to that of the homologs $\mathrm{Zr}$ and $\mathrm{Hf}$ [20]. This pilot experiment demonstrated that the SISAK system in combination with liquid scintillation detectors and a physical pre-separator can be used for future investigations of chemical properties of transactinide elements.

\section{Element 105, dubnium (Db)}

First studies of the aqueous-phase chemistry of $\mathrm{Db}$ were conducted manually using 34-s ${ }^{262} \mathrm{Db}$ produced in the irradiation of ${ }^{249} \mathrm{Bk}$ with ${ }^{18} \mathrm{O}$ ions at the LBNL 88-inch cyclotron [21]. Like the homologs niobium $(\mathrm{Nb})$ and tantalum $(\mathrm{Ta}), \mathrm{Db}$ was adsorbed on a glass surface upon fuming with nitric acid. The other chemical separation attempted in [21] involved the extraction of anionic fluoride species into methyl isobutyl ketone (MIBK). Under these conditions, Ta was extracted into MIBK nearly quantitatively, while $\mathrm{Nb}$ was extracted to only a small extent. It was found that $\mathrm{Db}$ is not behaving chemically like its lighter homolog Ta. The non Ta-like behaviour of $\mathrm{Db}$ might indicate that $\mathrm{Db}$ forms polynegative anions like $\left[\mathrm{DbF}_{7}\right]^{2-}$ under the chosen conditions.

To investigate this unexpected finding and more facets of the Db chemistry, a large number of automated separations were conducted with ARCA II. Here, we describe typical results from reversed-phase extraction chromatography of $\mathrm{Db}$. In the first experiments, separations with the liquid anion-exchanger triisooctyl amine (TiOA) on an inert support were performed [22]. It was found that TiOA extracts all group-5 elements including the pseudo-homolog protactinium $(\mathrm{Pa})$ from $\mathrm{HCl}$ solutions above $10 \mathrm{M}$. At lower $\mathrm{HCl}$ concentration, however, Db showed a striking non Ta-like behaviour and it followed its lighter homolog $\mathrm{Nb}$ and $\mathrm{Pa}$. From this similarity, it was concluded that the complex structure of 

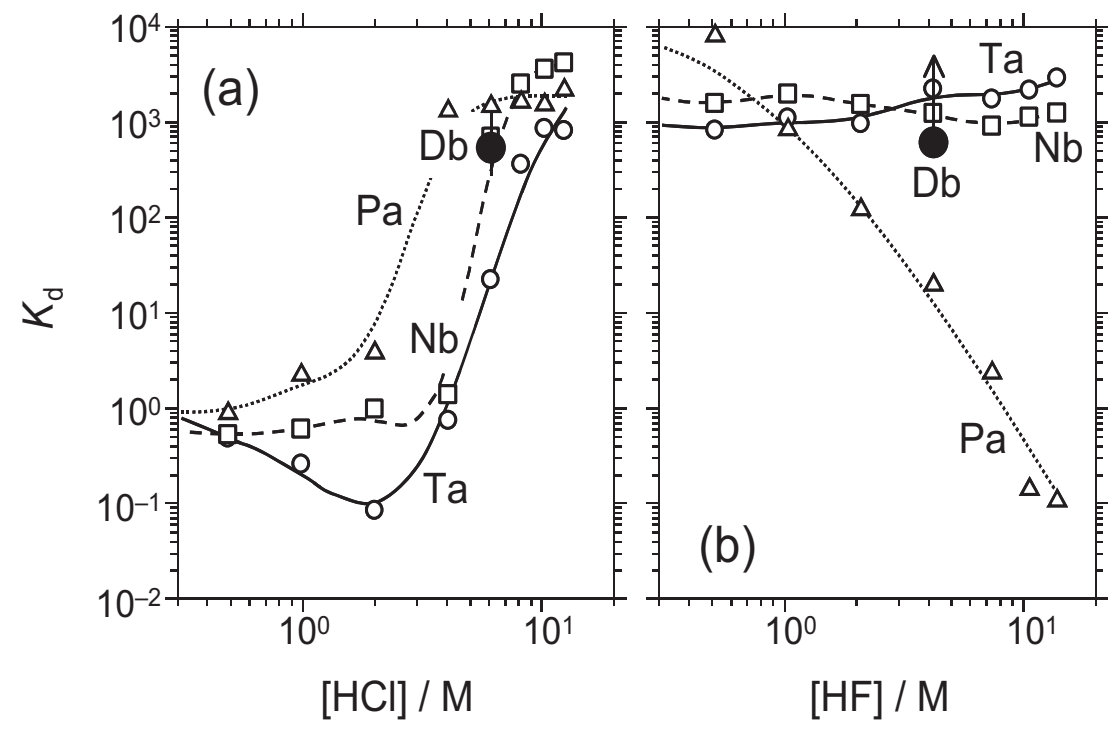

Figure 2. (a) Variation of the $K_{\mathrm{d}}$ values $\left(\mathrm{mL} \cdot \mathrm{g}^{-1}\right)$ of $\mathrm{Nb}, \mathrm{Ta}$, and $\mathrm{Pa}$ from $\mathrm{HCl}$ solutions into Aliquat $336 / \mathrm{HCl}$. The $K_{\mathrm{d}}$ value of $\mathrm{Db}$ in $6 \mathrm{M} \mathrm{HCl}$ (with error bars encompassing the $68 \%$ confidence limit) is indicated by the bold dot. (b) Extraction behaviour of $\mathrm{Nb}$, Ta, and Pa from HF solutions in Aliquat $336 / \mathrm{HF}$. The lower limit for the $K_{\mathrm{d}}$ value of $\mathrm{Db}$ in $4 \mathrm{M} \mathrm{HF}$ (representing the $68 \%$ confidence limit) is indicated by the bold dot with the arrow. Adapted from [24].

Db was $\left[\mathrm{DbOCl}_{4}\right]^{-}$or $\left[\mathrm{Db}(\mathrm{OH})_{2} \mathrm{Cl}_{4}\right]^{-}$. In addition, the preferential formation of oxyhalide complexes of $\mathrm{Db}$ was predicted theoretically [23].

Then, amine extraction experiments with Aliquat 336 (trioctyl-methylammonium chloride) of group-5 elements were systematically performed [24]. Chromatographic column separations with ARCA II were developed to study separately the fluoride and chloride complexation of $\mathrm{Db}$. In the system Aliquat $336 / \mathrm{HCl}$, a $K_{\mathrm{d}}$ value of $438_{-166}^{+532} \mathrm{~mL} \cdot \mathrm{g}^{-1}$ for $\mathrm{Db}$ in $6 \mathrm{M} \mathrm{HCl}$ was deduced, which is close to that of $\mathrm{Nb}$ and differs from the values for $\mathrm{Pa}$ and Ta; see Fig. 2a. Thus, the extraction sequence $\mathrm{Pa}>\mathrm{Nb} \geq \mathrm{Db}>\mathrm{Ta}$ is established exactly as theoretically predicted [25]. In the system Aliquat 336/HF, the result is depicted in Fig. 2b. The interpretation of these results indicates that the amine extraction behaviour of $\mathrm{Db}$ halide complexes is similar to that of its lighter homolog $\mathrm{Nb}$. In HF solution, the behaviour of $\mathrm{Db}$ differs clearly from that of $\mathrm{Pa}$, while in $\mathrm{HCl}$ it differs considerably from $\mathrm{Ta}$.

The anion-exchange behaviour of Db produced in the ${ }^{248} \mathrm{Cm}\left({ }^{19} \mathrm{~F}, 5 n\right){ }^{262} \mathrm{Db}$ reaction at the JAEA tandem accelerator was investigated together with $\mathrm{Nb}$, Ta, and $\mathrm{Pa}$ in mixed $\mathrm{HF} / \mathrm{HNO}_{3}$ solution [26]. The $K_{\mathrm{d}}$ value of $\mathrm{Db}$ in $0.31 \mathrm{M} \mathrm{HF} / 0.10 \mathrm{M} \mathrm{HNO}_{3}$ solution $\left(\left[\mathrm{F}^{-}\right]=3 \times 10^{-3} \mathrm{M}\right)$ was measured, where $\mathrm{Nb}$ and $\mathrm{Ta}$ form $\left[\mathrm{NbOF}_{4}\right]^{-}$and $\left[\mathrm{TaF}_{6}\right]^{-}$, respectively. It was found that the adsorption of $\mathrm{Db}$ on the resin is considerably weaker than that of $\mathrm{Ta}$ and it is similar to that of $\mathrm{Nb}$ and $\mathrm{Pa}$. This result suggests that $\mathrm{Db}$ forms a fluoro-oxo complex $\left[\mathrm{DbOF}_{4}\right]^{-}$, like $\mathrm{Nb}$, but not $\left[\mathrm{DbF}_{6}\right]^{-}$, like Ta. Note that the $K_{\mathrm{d}}$ value of $\mathrm{Db}$ is also close to that of $\mathrm{Pa}$ that forms $\left[\mathrm{PaOF}_{5}\right]^{2-}$ and/or $\left[\mathrm{PaF}_{7}\right]^{2-}$. The formation of complexes such as $\left[\mathrm{DbOF}_{5}\right]^{2-}$ and $\left[\mathrm{DbF}_{7}\right]^{2-}$ could also be suggested for $\mathrm{Db}$. To unequivocally clarify the fluoride complexation of $\mathrm{Db}$, further systematic studies of the $\mathrm{Db}$ behaviour as a function of $\left[\mathrm{F}^{-}\right]$and $\left[\mathrm{NO}_{3}^{-}\right]$ are required. Presently available results from studies in various aqueous solutions indicate 
that $\mathrm{Db}$ behaves more like the homolog $\mathrm{Nb}$, sometimes like its pseudo-homolog $\mathrm{Pa}$, but not like Ta.

As discussed in [24], the extraction sequences in Figs. $2 \mathrm{a}$ and $2 \mathrm{~b}$ are in good agreement with the theoretical calculations in [25] and [27], respectively. Although the non-Ta behaviour of $\mathrm{Db}$ in [26] is qualitatively consistent with the calculation [27], we need further investigations, such as determination of a chemical species of $\mathrm{Db}$, for a quantitative interpretation.

\section{Perspectives}

The first chemical separation and characterization of element 106 , seaborgium $(\mathrm{Sg})$, in the aqueous phase was performed using ${ }^{265} \mathrm{Sg}\left(T_{1 / 2}=7.4 \mathrm{~s}\right)$ produced in the reaction of ${ }^{22} \mathrm{Ne}$ with ${ }^{248} \mathrm{Cm}$ at the UNILAC (UNIversal Linear Accelerator) at the GSI (Gesellschaft für Schwerionenforschung) [28, 29]. (Today's knowledge on the decay of ${ }^{265} \mathrm{Sg}$ shows the presence of two states; one with a half-life of about $9 \mathrm{~s}$ and one with about $15 \mathrm{~s}$ [30]). 3900 identical cation-exchange separations in $0.1 \mathrm{M} \mathrm{HNO}_{3} / 5 \times 10^{-4} \mathrm{M}$ HF were performed with ARCA II. Three correlated mother-daughter $(\alpha-\alpha)$ decays were observed that were assigned to the decay of ${ }^{261} \mathrm{Rf}$ and ${ }^{257} \mathrm{No}$, as the descendants of ${ }^{265} \mathrm{Sg}$. The mother decays were not observed because it presumably decayed in the time interval between the end-of-separation and the start-of-measurement. However, it was suggested that $\mathrm{Sg}$ behaves like a typical group-6 element and forms neutral or anionic oxide or oxide halide compounds; in contrast to its pseudo-homolog uranium (U). Further investigation with a newly developed continuouslyworking system, however, is required to clarify the properties of $\mathrm{Sg}$ as mentioned below.

New technical and methodological developments have been carried out and are still ongoing, which provide a basis for future aqueous-phase chemistry experiments. These have the potential to provide new insights into properties of the lighter transactinides and to push the frontier even beyond $\mathrm{Sg}$. Successive complex formation experiments with $\mathrm{Sg}$ and beyond are most fascinating. Another option is to perform electrochemical studies of transactinide elements using redox reactions. Advanced theoretical model calculations have already provided quantitative predictions [31]. Preparations have started to measure the redox potential of hexavalent Sg [32]; a complex and continuously working system is envisioned which consists of an electrochemistry apparatus $[33,34]$ for the potentially controlled $\mathrm{Sg}$ reduction connected to a separation device like SISAK for the extraction and a flow-through liquid-scintillation detection system [35]. By means of a newly designed interface [36], this shall be coupled with a recoil separator like GARIS (Gas-filled Recoil Ion Separator) at RIKEN [37]. In this future program, the ${ }^{265} \mathrm{Sg}$ nuclide produced in the ${ }^{248} \mathrm{Cm}\left({ }^{22} \mathrm{Ne}\right.$, $5 n$ ) reaction is planned to be pre-separated from the primary beam and nuclear reaction by-products by GARIS, followed by thermalization in a stopping-gas cell and transfer to the chemistry set-up in an aerosol loaded gas-jet system. The products are fed into the electrochemistry apparatus to reduce the $\mathrm{Sg}^{6+}$ to the presumably most likely tetravalent state. Then, ions in the hexavalent cationic and in a low oxidation states are separated from each other by solvent extraction in an automated apparatus like SISAK. Both streams of liquid will be assayed by $\alpha$ spectrometry. If successful, this will give the first information on redox properties of a transactinide element.

Interesting perspectives arise from the observation of relatively long-lived isotopes of all elements from element 108, hassium (Hs) to element 114, flerovium (Fl), first observed in the pioneering works at the Flerov Laboratory of Nuclear Reactions (FLNR) [38]. Mostly synthesized in ${ }^{48} \mathrm{Ca}$-induced reactions $\left({ }^{26} \mathrm{Mg}\right.$ in the case of ${ }^{269-271} \mathrm{Hs}$ [39]) not only these directly produced isotopes are of interest but also the $\alpha$-decay grand-grand-daughter nuclei 
in the Rf to Db region, some having half-lives of the order of one day. On the one hand, this makes chemical investigations of the heaviest element in the Periodic Table all the way up to Fl conceivable. The chemistry of these elements is extremely interesting due to the predicted dramatic influence of relativistic effects [40]. In addition, being able to investigate isotopes of $\mathrm{Rf}$ and $\mathrm{Db}$ with half-lives as long as a day opens up new perspectives for the application of new techniques which require longer times but provide deeper insights. However, for all these studies at first new technical developments are mandatory to cope with cross section in the range between $10 \mathrm{pb}$ and $1 \mathrm{pb}$.

\section{References}

[1] M. Schädel, Angew. Chem. Int. Ed. 45, 368 (2006).

[2] J.V. Kratz, Handbook of Nuclear Chemistry, 2nd ed., vol. 2, eds. A. Vértes, S. Nagy, Z. Klencsár, R.G. Lovas, F. Rösche (Springer, Dordrecht, 2011), p. 925.

[3] M. Schädel, Radiochim. Acta 100, 579 (2012).

[4] A. Türler and V. Pershina, Chem. Rev. 113, 1237 (2013).

[5] M. Schädel and D. Shaughnessy, The Chemistry of Superheavy Elements, 2nd edition (Springer, Berlin, 2014).

[6] M. Schädel, Phil. Trans. R. Soc. A 373, 20140191 (2015).

[7] J.V. Kratz, Radiochim. Acta 99, 477 (2011).

[8] J.V. Kratz and Y. Nagame, Liquid-Phase Chemistry of Superheavy Elements. In The Chemistry of Superheavy Elements. 2nd edition, eds. M. Schädel and D. Shaughnessy (Berlin, Springer, 2014), p. 309.

[9] Y. Nagame et al., J. Radioanal. Nucl. Chem. 300, 77 (2014).

[10] Y. Nagame, J.V. Kratz, M. Schädel, Nucl. Phys. A 944, 614 (2015).

[11] M. Schädel et al., Radiochim. Acta 48, 171 (1989).

[12] Y. Nagame et al., Radiochim. Acta 93, 519 (2005).

[13] R.J. Silva et al., Inorg. Nucl. Chem. Lett. 6, 871 (1970).

[14] E.K. Hulet et al., J. Inorg. Nucl. Chem. 42, 79 (1980).

[15] E. Strub et al., Radiochim. Acta 88, 265 (2000).

[16] Y. Ishii et al., Bull. Chem. Soc. Jpn 84, 903 (2011).

[17] A. Toyoshima et al., Radiochim. Acta 96, 125 (2008).

[18] H. Haba et al., J. Am. Chem. Soc. 126, 5219 (2004).

[19] V. Pershina et al., Radiochim. Acta 90, 869 (2002).

[20] J.P. Omtvedt et al., J. Nucl. Radiochem. Sci. 3, 121 (2002).

[21] K.E. Gregorich et al., Radiochim. Acta 43, 223 (1988).

[22] J.V. Kratz et al., Radiochim. Acta 48, 121 (1989).

[23] V. Pershina et al., Radiochim. Acta 64, 37 (1994).

[24] W. Paulus et al., Radiochim. Acta 84, 69 (1999).

[25] V. Pershina, Radiochim. Acta 80, 75 (1998).

[26] Y. Kasamatsu et al., Chem. Lett. 38, 1084 (2009).

[27] V. Pershina and T. Bastug, Radiochim. Acta 84, 79 (1999).

[28] M. Schädel et al., Nature 388, 55 (1997).

[29] M. Schädel et al., Radiochim. Acta 83, 163 (1998).

[30] H. Haba et al., Phys. Rev. C 85, 024611 (2012).

[31] V. Pershina, E. Johnson, B. Fricke, J. Phys. Chem. A 103, 8463 (1999).

[32] A. Toyoshima et al., J. Radioanal. Nucl. Chem. 303, 1169 (2015).

[33] A. Toyoshima et al., Radiochim. Acta 96, 323 (2008).

[34] A. Toyoshima et al., Inorg. Chem. 52, 12311 (2013). 
[35] L. Stavsetra et al., Nucl. Instr. Meth. A 543, 509 (2005).

[36] K. Ooe et al., J. Radioanal. Nucl. Chem. 303, 1317 (2015).

[37] H. Haba et al., Chem. Lett. 38, 426 (2009).

[38] Yu.Ts. Oganessian, V.K. Utyonkov, Nucl. Phys. A 944, 62 (2015).

[39] J. Dvorak et al., Phys. Rev. Lett. 97, 242501 (2006).

[40] V. Pershina, Theoretical Chemistry of the Heaviest Elements. In The Chemistry of Superheavy Elements. 2nd edition, eds. M. Schädel and D. Shaughnessy (Berlin, Springer, 2014), p. 135. 